This manuscript is a preprint and has been submitted for publication in Remote Sensing of Environment. Please note that, despite having undergone peer-review, the manuscript has yet to be formally accepted for publication. Subsequent versions of this manuscript may have slightly different content. If accepted, the final version of this manuscript will be available via the 'Peer-reviewed Publication DOI' link on the right-hand side of this webpage. Please feel free to contact any of the authors; we welcome feedback. 


\title{
Automatic atmospheric correction for shortwave hyperspectral remote sensing data using a time-dependent deep neural network
}

\author{
Jian Sun ${ }^{\mathrm{a}, \mathrm{b}, \mathrm{d}, *}$, Fangcao Xu ${ }^{\mathrm{a}, \mathrm{d}}$, Guido Cervone ${ }^{\mathrm{a}, \mathrm{c}, \mathrm{d}}$, Melissa Gervais ${ }^{\mathrm{c}, \mathrm{d}}$, Christelle Wauthier ${ }^{\mathrm{b}, \mathrm{d}}$, Mark Salvador ${ }^{\mathrm{e}}$ \\ ${ }^{a}$ Department of Geography, The Pennsylvania State University, University Park, PA, USA \\ ${ }^{b}$ Department of Geoscience, The Pennsylvania State University, University Park, PA, USA \\ ${ }^{c}$ Department of Meteorology and Atmosphere Science, The Pennsylvania State University, University Park, PA, USA \\ ${ }^{d}$ Institute for Computational and Data Sciences, The Pennsylvania State University, University Park, PA, USA \\ ${ }^{e}$ Zi Inc, Washington D.C., USA
}

\begin{abstract}
Atmospheric correction is an essential step in hyperspectral imaging and target detection from spectrometer remote sensing data. State-of-the-art atmospheric correction algorithms either require filed-measurements or prior knowledge of atmospheric characteristics to improve the predicted accuracy, which are computational expensive and unsuitable for real time application. In this paper, we propose a time-dependent neural network for automatic atmospheric correction and target detection using multi-scan hyperspectral data under different elevation angles. Results show that the proposed network has the capacity to accurately provide atmospheric characteristics and estimate precise reflectivity spectra for different materials, including vegetation, sea ice, and ocean. In addition, experiments are designed to investigate the time dependency of the proposed network. The error analysis confirms that our proposed network is capable of estimating atmospheric characteristics under both hourly and diurnally varying environments. Both the predicted results and error analysis are promising and demonstrate that our network has the ability of providing accurate atmospheric correction and target detection in real-time.
\end{abstract}

Keywords: hyperspectral, deep learning, atmospheric correction, neural network, reflectivity

\section{Introduction}

The electromagnetic radiation characteristics of a material's absorption and emission are determined by its unique 3 molecular composition and texture. This provides an opportunity to obtain information necessary for identifying 4 objects using remote sensing techniques, instead of field measurements. One of such technique is hyperspectral 5 imaging (HSI), which has been proven to be a powerful tool to identify any given object of interest on the surface 6 of the Earth, without any direct physical contact, by retrieving their unique spectral signatures (Adão et al., 2017,

${ }^{*}$ Corresponding author

Email address: jbs6371@psu.edu (Jian Sun) 
Ghamisi et al., 2017, Manolakis et al., 2019). With recent developments in imaging spectroscopy, vast quantities of spectrometer data with rich spectral, spatial, and temporal information have been collected by sensors from airborne and spaceborne platforms (Teke et al., 2013). The collection of hyperspectral remote sensing data is deeply affected by the absorption and scattering of the atmosphere. For instance, atmospheric water vapor is efficient at absorbing incoming solar radiation in bands centered at approximately $0.94,1.14,1.38,1.88$, and $2.7 \mu \mathrm{m}$. Only approximately half of the 0.4-2.5 $\mu \mathrm{m}$ spectrum can be transmitted without absorption from atmospheric gases (Gao et al., 2006). In addition, the shorter wavelength spectral region below $1 \mu \mathrm{m}$ is also affected by molecular and aerosol scattering. Thus, to study the spectral properties of the Earth and retrieve its reflectivity, atmospheric effects must be removed from the spectrometer data.

Atmospheric correction algorithms for hyperspectral imaging data are generally divided into two major classes based on whether any physical mechanisms of solar transportation are involved, in which delineates the interaction between environment and surface. The first class consists of scene-based empirical methods, such as internal average reflectance (Kruse, 1988, or IAR), flat-field correction (Roberts et al., 1986), and empirical line approach (Conel et al. 1987, or ELA), which are computationally efficient and can be accurate with sufficient information from field measurements. However, these approaches either may cause unrealistic absorption features in the corrected reflectance spectra because of their robust assumptions (Clark and King, 1987; DiStasio Jr and Resmini, 2010), or require a prior knowledge of in-scene elements, i.e., field-measured reflectance spectra, for at least one bright target and one dark target which does not adapts for some scenarios (Conel et al., 1987, Aspinall et al., 2002).

q The other class of atmospheric correction algorithms are radiative transfer approaches (Gao et al., 1993, Gao and Davis, 1997; Adler-Golden et al., 1999; Siewert, 2000; Mayer, 2009; Duan et al., 2010) that take advantage of the known physical mechanisms of interactions between environments and surface targets. For instance, with an assumption of a simple homogeneous layer of atmosphere, the Pstar computational model was built to provide a discrete ordinate solution that only works under an optically-thin atmospheric condition (Siewert, 2000). The MYSTIC code is optimized using a Monte-Carlo algorithm that allows a more complex heterogeneous atmospheric profile and provides the solution with a high accuracy, however, its implementation is much more computational expensive and may generate statistical noise (Emde et al., 2010, Mayer, 2009). The SOSVRT radiative transfer model uses successive-order-scattering (SOS) method that leads to improvement in modeling atmospheric scattering, however challenges remain in its ability to converge when the atmosphere is the optically-thick and the observing angles are oblique (Duan et al., 2010). These first-principle approaches are capable of retrieving high accuracy reflectance spectra with prior knowledge of atmospheric characteristics by modeling the absorption and scattering effects of atmosphere. However, the explicit atmospheric spectra simulations are not only computationally expensive, but require accurate 
characterization of the atmosphere and precise characteristics of sensors (Siewert, 2000, Qu et al., 2003, Gao et al. 2009).

The combination of empirical methods and radiative transfer approach have also been proposed to accelerate the process of atmospheric correction for multi-channel hyperspectral remote sensing data (Qu et al., 2003, Gao et al., 2009; Markelin et al., 2017, Katkovsky et al., 2018), however, its performance is a trade-off between computational efficiency and accuracy. Thus, an accurate, fast, and automated atmospheric correction approach is desired to retrieve the emitted and reflected radiance of the target at the Earth's surface from the hyperspectral remote sensing data.

Recent advances in agile collection platforms overcome the fixed nadir looking geometry settings of past instruments, which allow users to measure additional solar and atmospheric radiative components of the radiative transfer equation (RTE). Additionally, the new generation of agile sensors have the ability to revisit a scene in seconds under variant looking angles. The rapid multi-scan collecting capacity and the geometric diversity of modern hyperspectral collecting systems provide a new opportunity to develop a more complete solution to the fundamental RTE because of their retrieval of full spatial dimensionality and multiple temporal components. Furthermore, the abundant spectralspatial information in multi-scan hyperspectral remote sensing data enables the adoption of the fully data-driven methods, such as deep learning technique, in hyperspectral image analysis.

Deep learning technique, as a branch of machine learning and artificial intelligence methods, has shown research and operational success across various fields. A representative framework of deep learning is the deep neural network (DNN). For image processing applications, the convolution neural network (CNN) has demonstrated its superiority since it can be formulated with a relatively small number of trainable parameters. Various architectures of CNN have been designed for different imaging tasks such as AlexNet (Krizhevsky et al., 2012), VGG-16 (Simonyan and Zisserman, 2014), U-Net (Ronneberger et al., 2015), Inception (Szegedy et al., 2015, 2016), ResNet (He et al., 2016), and DenseNet (Huang et al., 2017). Beyond that, progresses of applying CNN in discipline of hyperspectral image analysis have also been made. For instance, Yu et al. (2017) demonstrated the hyperspectral image classification using a CNN outperforms other state-of-the-art methods. Xu et al. (2020) proposed an encoder-decoder neural network to remove atmospheric effects from long wavelength multi-scan hyperspectral data. These studies have shown the potential use on DNNs in its application to hyperspectral image analysis. However, seasonal and diurnal cycle variations of atmosphere were not taken into account in data collection, which limits the performance and feasibility of deep learning in some realistic scenarios. In addition, deep learning application using the shortwave spectrum of hyperspectral data remain in an unexplored area because of the complexity of the spectral-spatial nature.

In this paper, we design a time-dependent neural network to solve every radiative component of RTE from the atsensor total radiance using shortwave spectra. The proposed network is partially convolution-based, but involves two 
temporal factors, i.e., collection day of the year and time of the day, using the fully connected layers. We expect the time-dependency of such a neural network to capture the diurnally-and-hourly varying characteristics of atmospheric environment, which can improve the accuracy of estimating the target's reflectivity spectra. This paper is organized as follows: first, we introduce the theoretical deep learning solution of the RTE and discuss the architectural design of the time-dependent neural network; second, we describe the hyperspectral data simulation, the required pre-processing of input and labelled data, and the network training process. The performance of our designed neural network is then evaluated by analyzing the discrepancy between the ground-truth solar and atmospheric radiative components of RTE and the predicted results. The target's reflectivity is retrieved as an additional indicator to evaluate the network's ability. Finally, the contribution of the two temporal factors and the composition of the training dataset to the network's prediction skill are also discussed.

\section{Methodology}

In this section, we first briefly review the concepts of radiative transfer modeling, and different components of the RTE. Then, the fully-data driven deep learning solution of the RTE will be introduced following by the discussion of the neural network architecture to adapt for the diurnal and hourly variations of atmospheric conditions.

\subsection{Radiative transfer modeling}

In addition to the chemical and physical compositions of the target, the hyperspectral remote sensing data is also highly affected by the solar-sensor's geometry settings and the absorption and scattering by atmosphere, including gases, aerosols, water vapor, and clouds. The physical transportation of radiative energy can be described by the RTE, and its mathematical expression (Schott, 2007) is written as

$$
\begin{aligned}
L_{\lambda}= & \frac{\cos \sigma}{\pi} E_{s \lambda} r(\lambda) \tau_{1}(\lambda) \tau_{2}(\lambda)+L_{T \lambda} \varepsilon(\lambda) \tau_{2}(\lambda)+L_{u s \lambda}+L_{u \epsilon \lambda} \\
& +\left[F\left(L_{d s \lambda}+L_{d \epsilon \lambda}\right)+(1-F)\left(L_{b s \lambda}+L_{b \epsilon \lambda}\right)\right] r(\lambda) \tau_{2}(\lambda)
\end{aligned}
$$

where $T$ is the target's temperature in Kelvin, $s$ represents that the component is solar-related, $\epsilon$ denotes the selfemitted thermal component, $d(u)$ is the downwelling (upwelling) component, and $b$ is a reminder that the component pertains to the background. $F$ is the fraction of hemisphere is obscured by background objects, and is also known as the shape factor. $\lambda$ denotes the wavelength of the solar radiance. The physical meaning of each RTE component is listed in Table 1. 
Table 1: Physical meaning of each component in RTE

\begin{tabular}{l|l|l}
\hline Components & Units & Physical meaning \\
\hline$E_{s \lambda}$ & $W / m^{2}$ & Extraterrestrial solar irradiance \\
$\sigma$ & - & Incident angle of solar irradiance \\
$r(\lambda)$ & - & Spectral reflectivity of the target \\
$\varepsilon(\lambda)$ & - & Spectral emittance of the target \\
$\tau_{1}(\lambda)$ & - & Atmospheric transmission on the sun-target path \\
$\tau_{2}(\lambda)$ & - & Atmospheric transmission on the target-sensor path \\
$L_{T \lambda}$ & $W c m^{-2} s r^{-1} \mu \mathrm{m}^{-1}$ & Spectral radiance of a blackbody at temperature $T$ \\
$L_{d s \lambda}+L_{d \epsilon \lambda}$ & $W c m^{-2} s r^{-1} \mu \mathrm{m}^{-1}$ & Sum of solar and atmospheric downwelling spectral radiance \\
$L_{b s \lambda}+L_{b \epsilon \lambda}$ & $W c m^{-2} s r^{-1} \mu \mathrm{m}^{-1}$ & Sum of background reflected and self-emitted spectral radiance \\
$L_{u s \lambda}+L_{u \epsilon \lambda}$ & $W c m^{-2} s r^{-1} \mu \mathrm{m}^{-1}$ & Sum of solar and atmospheric upwelling spectral radiance \\
\hline
\end{tabular}

In Table 1, $L_{T \lambda}$ is the emitted radiance of a blackbody, also referred to as the Planck's Law:

$$
L_{T \lambda}=2 h c^{2} \lambda^{-5}\left(e^{\frac{h c}{\lambda k T}}-1\right)^{-1}
$$

wh

where, $h$ is Planck's constant, $c$ is the speed of light, and $k$ is the Boltzmann gas constant. To achieve energy balance all incident flux must be either transmitted, reflected, or absorbed, i.e., $\tau(\lambda)+r(\lambda)+\alpha(\lambda)=1$. Thus, the spectral emittance of an opaque surface can be calculated by its reflected radiance: $\varepsilon(\lambda)=1-r(\lambda)$ because of its zero transmissivity (i.e., $\tau(\lambda)=0$ ) and the equivalence of the absorptivity and the emissivity (i.e., $\alpha(\lambda) \equiv \varepsilon(\lambda)$ ). Assuming the object of interest is located in an open area, i.e., $F=1$, Equation (1) can be reformulated as

$$
\begin{aligned}
L_{\lambda} & =\tau_{2}(\lambda) r(\lambda)\left[\frac{\cos \sigma}{\pi} E_{s \lambda} \tau_{1}(\lambda)+L_{d s \lambda}+L_{d \epsilon \lambda}\right]+\varepsilon(\lambda) \tau_{2}(\lambda) L_{T \lambda}+L_{u s \lambda}+L_{u \epsilon \lambda} \\
& =\tau_{2}(\lambda) r(\lambda) L_{\text {down }}^{M}+[1-r(\lambda)] \tau_{2}(\lambda) L_{T \lambda}+L_{\text {solar_scat } 1}^{M}+L_{\text {solar_scat } 2}^{M}+L_{\text {path_emit }}^{M}+L_{\text {path_scat }}^{M}
\end{aligned}
$$

where, $L_{d o w n}^{M}=\left[\frac{\cos \sigma}{\pi} E_{s \lambda} \tau_{1}(\lambda)+L_{d s \lambda}+L_{d \epsilon \lambda}\right]$ describes the downwelling radiance in which the ground reflected radiance received at the sensor can be delineated as $\tau_{2}(\lambda) r(\lambda) L_{\text {down }}^{M}$; The solar scattering components are $L_{u s \lambda}=$ $L_{\text {solar_scat } 1}^{M}+L_{\text {solar_scat } 2}^{M}$ where the right hand of equation $L_{\text {solar_scat } 1}^{M}$ and $L_{\text {solar_scat } 2}^{M}$ represent the single- and multiplescattering solar radiances, respectively; and $L_{u \epsilon \lambda}=L_{\text {path_emit }}^{M}+L_{\text {path_scat }}^{M}$ demonstrates atmospheric thermal radiance in and scattered to the light-of-sight (LOS) path.

\subsection{Deep learning solution of the RTE}

Instead of using a single viewpoint in traditional radiative transfer approaches, the multi-scan hyperspectral geometry settings allow users to re-evaluate the RTE using a fully data-driven deep learning technique. The desired input of such a neural network will be multi-scan multispectral remote sensing data collected in a rapid sequence. The high temporal scanning frequency allows us to consider atmospheric conditions to be constant for the multi-scan data 
collected at specific time and the multiple downlooking angles circumvents the underdetermined problem. The aim of the neural network is to study all atmospheric characteristics that affect the total radiance observed by the sensors. In other words, an applicable network should be capable of retrieving all solar and atmospheric coherent radiative components of the RTE, including $\tau_{2}(\lambda), L_{\text {down }}^{M}, L_{\text {solar_scat } 1}^{M}, L_{\text {solar_scat } 2}^{M}, L_{\text {path_emit }}^{M}$, and $L_{\text {path_scat }}^{M}$, which will subsequently be referred to as the six solar and atmospheric RTE components. However, due to the rapidly changing environment and the varying incident angles between the Sun and target, it is still unrealistic to directly decompose the total radiance $L_{T \lambda}$ into all atmospheric components using a single equation without any prior knowledge, even with the assistance of the multi-scan hyperspectral data. To take into account the diurnal and hourly variability of atmosphere and their impacts on the incoming solar radiation and climatological atmospheric conditions, two temporal factors, day of the year and time of the day, need to be considered in the network design. With all these information having been correctly placed, the fundamental formulation of the desired neural network for atmospheric correction on hyperspectral data is written as

$$
\tau_{2}(\lambda), L_{\text {down }}^{M}, L_{\text {path_emit }}^{M}, L_{\text {path_scat }}^{M}, L_{\text {solar_scat } 1}^{M}, L_{\text {solar_scat } 2}^{M}=\Omega\left(L_{\lambda \theta}, \text { day, time }\right)
$$

where the label $\theta$ represents the elevation angle in the observation geometry, $L_{\lambda \theta}$ is the total radiance under multiple elevation angles received at a certain time and day by the sensor. $\Omega$ represents a sequence of input preprocessing, network implementation, and output postprocessing. After obtaining the final estimations using $\Omega$, the target's reflectivity can be calculated as

$$
r(\lambda)=\frac{\left(L_{\lambda \theta}-L_{u s \lambda}-L_{u \epsilon \lambda}\right) / \tau_{2}(\lambda)-L_{T \lambda}}{L_{d o w n}^{M}-L_{T \lambda}}
$$

\subsection{Network architecture}

Various neural network architectures have been developed for different types of tasks. Here, similarly to general image processing, we adopt the encoder-decoder $\mathrm{CNN}$ as the major framework for the atmospheric correction problem. However, as previously discussed, the encoder-decoder architecture needs an adjustment to incorporate the two temporal factors (i.e., two scalar values) to adapt to both the diurnal and variability in atmospheric conditions and the varying incident angles.

In Figure 1 we plot the architecture for our time-dependent neural network for atmospheric correction on multiscan hyperspectral data. There are three primary blocks including an encoder, the fully-connected layers in latent space, and a decoder. All detailed parameterizations in each layer are listed in Table 2 . Our neural network accepts a total radiance relieved at the sensor with 150 wavelength bands (height) from $0.4 \mu \mathrm{m}$ to $3.0 \mu \mathrm{m}$ under 13 elevation 


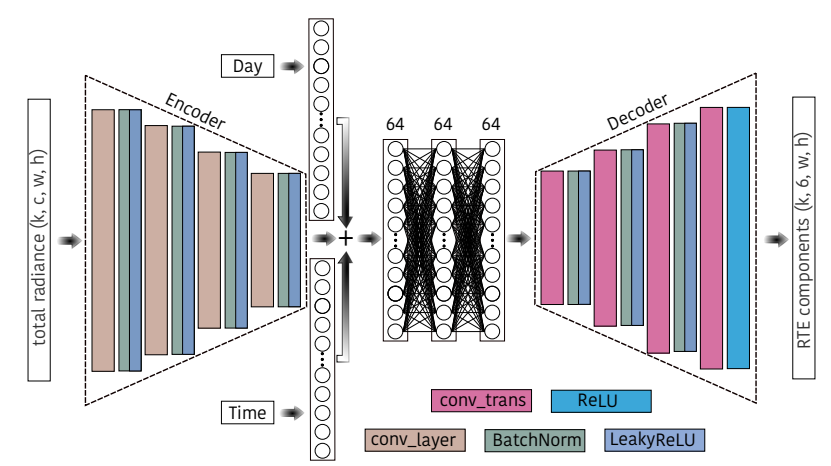

Figure 1: The architecture of the proposed time-dependent neural network.

Table 2: Detailed parameters of the proposed time-dependent CNN shown in Figure 1

\begin{tabular}{c|l|ccccc}
\hline \multicolumn{2}{c|}{ Operation Layer } & $\begin{array}{c}\text { Number } \\
\text { of Filers }\end{array}$ & $\begin{array}{c}\text { Size of } \\
\text { Each Filter }\end{array}$ & Stride & Padding & $\begin{array}{c}\text { Output Size } \\
\text { (c x h x w) }\end{array}$ \\
\hline Input & total radiance & - & - & - & - & $1 \times 150 \times 13$ \\
\hline \multirow{4}{*}{ Encoder } & Conv_layer_1 & 64 & $4 \times 3 \times 1$ & $2 \times 2$ & $1 \times 1$ & $64 \times 75 \times 7$ \\
\cline { 2 - 7 } & Conv_layer_2 & 128 & $5 \times 3 \times 64$ & $2 \times 2$ & $1 \times 1$ & $128 \times 37 \times 4$ \\
\cline { 2 - 7 } & Conv_layer_3 & 256 & $5 \times 3 \times 128$ & $2 \times 2$ & $1 \times 0$ & $256 \times 18 \times 1$ \\
\cline { 2 - 7 } & Conv_layer_4 & 64 & $18 \times 1 \times 256$ & $1 \times 1$ & $0 \times 0$ & $64 \times 1 \times 1$ \\
\hline \multirow{3}{*}{$\begin{array}{c}\text { Fully } \\
\text { Laynected }\end{array}$} & FC_day & - & - & - & - & 64 \\
\cline { 2 - 7 } & FC_time & - & - & - & - & 64 \\
\cline { 2 - 7 } & FC_1 & - & - & - & - & 64 \\
\cline { 2 - 7 } & FC_2 & - & - & - & - & 64 \\
\cline { 2 - 7 } & FC_3 & - & - & - & - & 64 \\
\hline \multirow{4}{*}{\begin{tabular}{c} 
Decoder \\
\cline { 1 - 7 }
\end{tabular}} & Conv_trans_1 & 256 & $18 \times 1 \times 64$ & $1 \times 1$ & $0 \times 0$ & $256 \times 18 \times 1$ \\
\cline { 2 - 7 } & Conv_trans_2 & 128 & $5 \times 4 \times 256$ & $2 \times 2$ & $1 \times 0$ & $128 \times 37 \times 4$ \\
\cline { 2 - 7 } & Conv_trans_3 & 64 & $5 \times 3 \times 128$ & $2 \times 2$ & $1 \times 1$ & $64 \times 75 \times 7$ \\
\cline { 2 - 7 } & Conv_trans_4 & 6 & $4 \times 3 \times 64$ & $2 \times 2$ & $1 \times 1$ & $6 \times 150 \times 13$ \\
\hline
\end{tabular}

The encoder in our architecture is designed to remove repetitive information from the multi-scan hyperspectral data by analyzing the invariance of the target's reflectivity with different elevation angles. To preserve the detailed information while reducing spatial dimensionality of the inputs, instead of using the max pooling layer, we adopt stride convolution in the encoder to compress the input into a latent space vector. The global convolutional filters with size of $18 \times 1 \times 256$ are applied in the last convolution layer of the encoder (see in Table 2 ) to avoid vast parameterizations in the latent space. Each convolution layer in the encoder is followed with a batch-normalization layer and a leaky rectifier unit (LeakyReLU) activation layer.

In the latent space, we use two independent fully connected layers to handle the two temporal factors: day of the year and time of the day, in which the outputs of these two layers are added into the encoder generated latent space vector. Then, three fully connected layers with 64 neurons in each are employed to decompose the summarized latent 
space vector into a RTE involved vector which will be expanded by the decoder into the same readable dimension as the input. A LeakyReLU transformation is carried out after every fully connected layer. Transposed convolutions are also applied as the upsampling strategy in the decoder, and a combination of a batch-normalization and a LeakyReLU are implemented after each transposed convolution layer, except for the last one which is followed by a ReLU activation. The outputs of the final ReLU layer are the solved six RTE components stored in different channels with the same order of as illustrated in the left hand of Equation (4).

\section{Numerical examples}

In this section, synthetic multi-scan hyperspectral data are simulated using MODTRAN (Adler-Golden et al., 1999. Berk et al. 2014) to train and validate the ability of the time-dependent neural network. After obtaining six predicted solar and atmospheric radiative components of the RTE using the trained network, the target's reflectivity can also be calculated using Equation (5) to further examine the performance of the proposed network.

\subsection{Data simulation}

MODTRAN software is chosen for synthetic hyperspectral data simulation. We assume that the target is located at $(40.7934 \mathrm{~N}, 77.86 \mathrm{~W})$ in an open area, i.e., the shape factor $F=1$, which lies at the Pennsylvania State University, University Park, PA, USA. These synthetic data were used to plan a data collection campaign, Nittany Radiance 2019, that occurred on the premises of the Pennsylvania State University, University Park campus on April 2019 to collect aerial hyperspectral remote sensing scenes as well as ground truth measurements. The at-sensor total radiance is collected by an airborne sensor with a constant range $5000 \mathrm{~m}$ and a fixed azimuth. The temperature of the target is set to $350 \mathrm{~K}$. In every data recording process, the total radiance is measured under 13 elevation angles starting from $30^{\circ}$ to $90^{\circ}$ at $5^{\circ}$ intervals. Additionally, for each target, the data is collected eight times a day from 6:00am to 8:00pm (represents local time subsequently) at 2 hours intervals all year.

The airborne sensor has a spectral resolution of $17.5 \mathrm{~nm}$ to record radiative signals at 150 wavelength bands between $0.4 \mu \mathrm{m}$ and $3.0 \mu \mathrm{m}$. With such a parameterization setting, for every material, we have $365 \times 8$ radiance maps of size $150 \times 13$. The simulations are performed on 48 target materials, including 42 MODTRAN builtin materials, and six opaque Lambertian gray bodies with different constant reflectivities across the spectrum, which are $0.05,0.1$, $0.3,0.5,0.8,1.0$

In Figure 2, the total radiances with 13 elevation angles for two different materials received at 2:00pm on January $10^{\text {th }}$ and their corresponding targets' reflectivity spectra are plotted. Figure 2 a shows the total radiance for a Lambertian gray with a 0.3 constant reflectivity shown in Figure $2 \mathrm{p}$. For the target with a constant reflectivity, prominent 

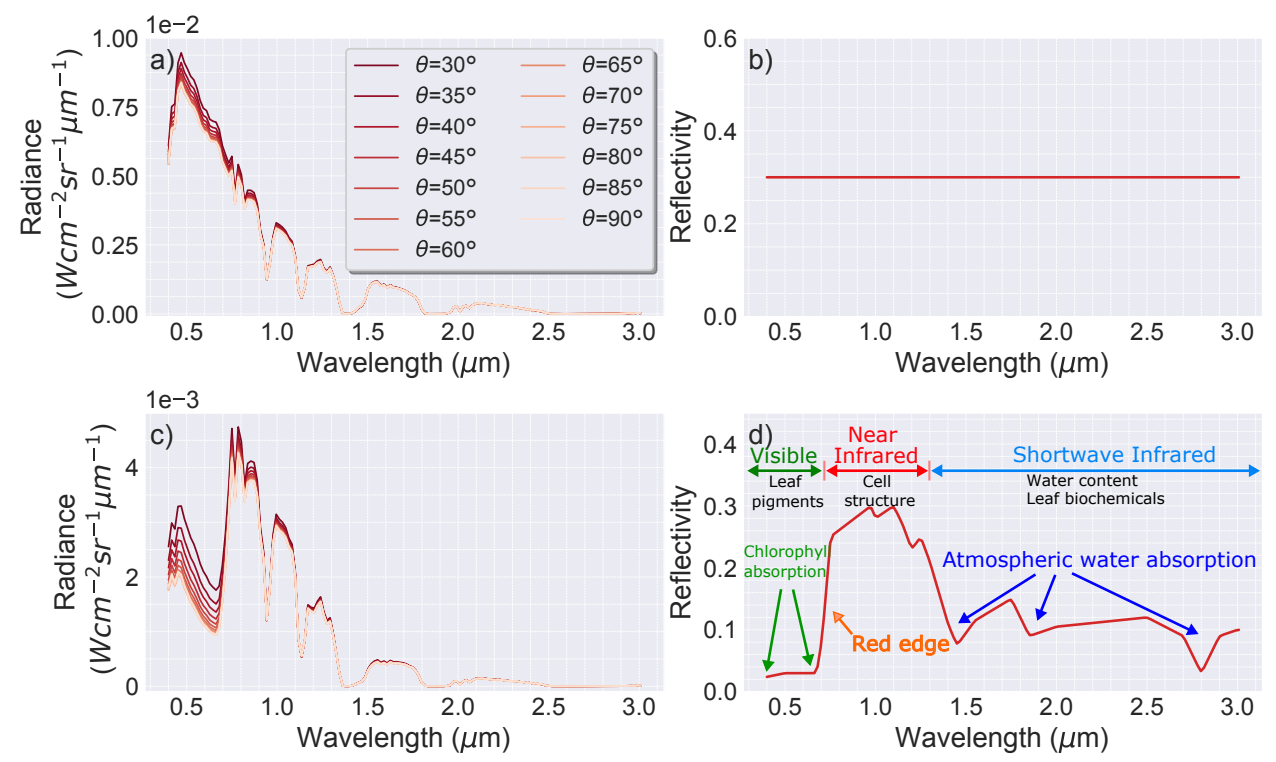

Figure 2: The at-sensor spectra for a Lambertian gray body with a 0.3 constant reflectivity and an evergreen needle forest received at 2:00pm on January $10^{\text {th }}$. a) the total radiance for the gray body, b) the gray body's reflectivity spectra, c) the total radiance for an evergreen needle forest, d) the reflectivity of the evergreen needle forest.

contributions of the elevation angle in sensor-received total radiance are only observed in the visible and near-infrared bands. The elevation angle's influence vanishes as the wavelength increases.

To study the elevation angle's influence on the total radiance for different materials, the simulated total radiances under 13 elevation angles for an evergreen needle forest collected at the same time and date (2:00pm on January $10^{\text {th }}$ ) are shown in Figure 2k, with the coherent target's reflectivity shown in Figure $2 \mathrm{~d}$. Compared to the total radiance spectrum for the gray body, the elevation angle caused distinction of the total radiance for the vegetation target manifests strongly in the visible spectrum, which is likely caused by the unique strong chlorophyll absorption. In addition, we find that, as the elevation angle increasing (i.e., shorter transmitting path in atmosphere), there is a decrease in the total radiance received at the sensor. This is due to the decrease in solar scattering with increasing elevation angle, as will be confirmed later in Figure 3 .

During the MODTRAN simulation, the six solar and atmospheric spectra are also preserved, which after preprocessing are considered as the ground-truth labeled data for the neural network. These six RTE radiative components for the evergreen needle forest are plotted in Figure 3 , and the corresponding total radiance is shown in Figure 2k. Figure 3 shows that atmospheric transmission is directly proportional to the elevation angle. There are three atmospheric complete absorption bands centered at $1.38 \mu \mathrm{m}, 1.88 \mu \mathrm{m}$, and $2.7 \mu \mathrm{m}$, respectively. As expected, Figure $3 \mathrm{p}$ shows that the elevation angle has no impact on the downwelling radiance. Figure $3 \mathrm{k}$ and $3 \mathrm{~d}$ show that atmosphere self-emitted thermal and scattering radiances are negligible in the visible and near-infrared regions. On the contrary, 
the solar scattering radiances are only observed in the visible and near-infrared regions shown in Figure 3 e and 3 .

Furthermore, the atmospheric emitted and scattering as well as the solar single and multiple scattering all decrease with increasing elevation angle (Figure 3 , d,e,f), consistent with the total radiance shown in Figure22.
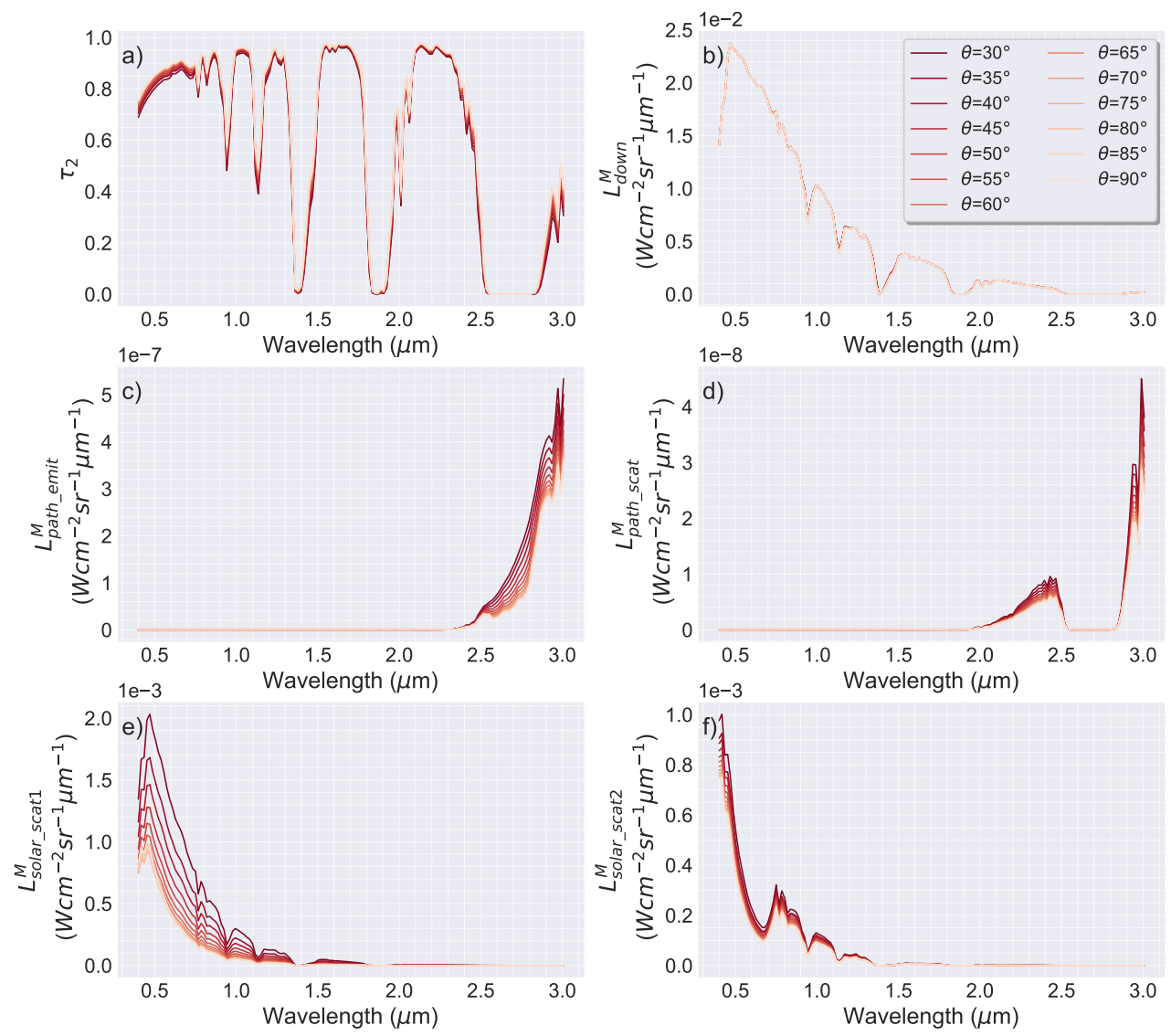

Figure 3: Six solar and atmospheric radiances for an evergreen needle forest simulated at 2:00pm on January 10 ${ }^{\text {th }}$. a) atmospheric transmission $\tau_{2}(\lambda)$, b) downwelling radiance $L_{d o w n}^{M}$, c) atmospheric self-emitted thermal radiance $L_{\text {path_emit }}^{M}$, d) atmospheric scattering radiance $L_{\text {path_scat }}^{M}$, e) solar single scattering radiance $L_{\text {solar_scat } 1}^{M}$, f) solar multiple scattering radiance $L_{\text {solar_scat } 2}^{M}$.

\subsection{Input and labeled data preparation}

Before feeding the simulated total radiance maps into the designed network with architecture shown in Figure 1 . we observe that the radiance dramatically decreases as the spectral band moves from the visible to the shortwave infrared region. For such a skewed input a large number of convolutional layers would be required to balance the contribution of different spectral bands and to reduce the risk of losing significant features in the shortwave infrared band due to the weight sharing in the CNN. Increasing the number of convolutional layers, however, not only incurs a of the network making it harder to train. 
An alternative approach is preprocessing the data to reduce the degrees of freedom of the network's input and output, while keeping the simplicity of the network. To do so, we calculated the Sun's blackbody spectrum at $5778 \mathrm{~K}$ using Planck's Law (Equation (2)) as shown in Figure 4. To preprocessing the total radiance, we divide the total radiance by the blackbody spectrum for every spectral band then multiply by a constant value of $10^{5}$ to re-scale the value into the range of $[0,1]$. This preprocessed data will subsequently be referred to as the normalized total radiance map or the input of the network. The total radiance maps and their normalization for a gray body with a constant reflectivity of 0.3 and an evergreen needle forest are shown in Figure 5 .

The maximum spectral values along every wavelength band and observation time are computed for each solar and atmospheric radiance to prepare the labeled data for the network's training stage. Each component is then divided by its corresponding maximum spectrum along each wavelength band and observation time. The final outputs of the network are six normalized solar and atmospheric radiance maps, which can be converted into the correct magnitude by multiplying by their corresponding maximum spectra at each wavelength and observation time. The matrix view of these six RTE components and their nomalizations are shown in Figure 6.

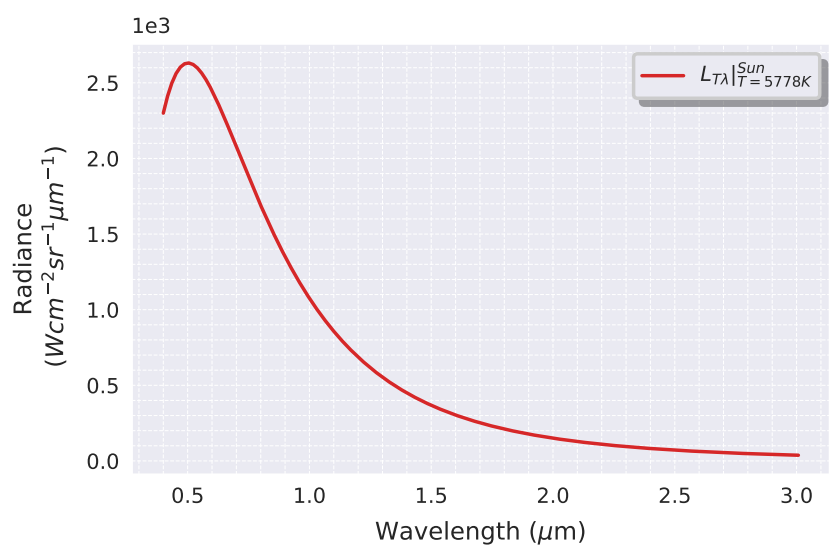

Figure 4: The spectrum of a blackbody at Sun's temperature 5778K.

\subsection{Training and validation}

The total radiance for 48 materials are simulated, which includes 42 MODTRAN builtin materials and six Lambertian gray bodies with different constant reflectivities. We chose hyperspectral simulations for 15 builtin vegetations and six gray bodies with varying constant reflectivities and randomly selected at different collecting time for each material as training $(80 \%)$ and validation $(20 \%)$ examples. The data simulated using the remaining 27 materials are treated as the test dataset. Thus, we have 49056 training examples in total, 12264 validation examples, and 78, 840 examples in the test dataset. During the training stage, an $\ell 2$-norm objective function is applied to measure the dis- 


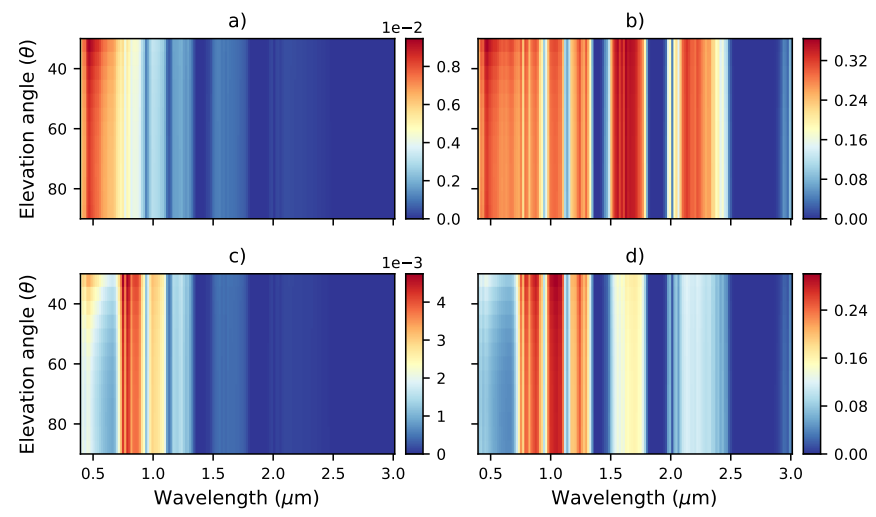

Figure 5: Matrix view of the total radiance and its normalization received at 2:00pm on January $10^{\text {th }}$. a) the total radiance for a Lambertian gray body with a 0.3 constant reflectivity. b) the normalized total radiance for the Lambertian gray body, c) the total radiance for an evergreen needle forest, d) the normalized total radiance for the evergreen needle forest.

crepancy between the output of the network and its corresponding normalized ground-truth. In addition, the adaptive momentum estimation, or Adam, algorithm (Kingma and Ba, 2014) with an initial learning rate $5 \times 10^{-4}$ is adopted to optimize all trainable parameters in the network by minimizing the objective function. The training process is implemented with 10 Nvidia Tesla P100-PCIe GPUs.

After training for 300 epochs, the network is evaluated using the simulated data for the 27 withheld materials from the test dataset. We applied the trained network to the evergreen needle forest extracted from the test dataset. The predicted results of six ground-truth solar and atmospheric radiances simulated at 2:00pm on January $10^{\text {th }}$ are plotted in Figure 7. From left to right in columns, they are six ground-truth atmospheric and solar radiances, predicted results using the proposed network, and the residuals of the first two columns, respectively. The results show that the residuals are at least one order of magnitude smaller than their corresponded ground-truth components. It is fair to say that the proposed network has the capacity of accurately estimating atmospheric characteristics from the atsensor total radiance with the two temporal factors. The instantaneous evaluation of the neural network provides an opportunity for real time atmospheric correction and target detection.

With the predicted six components shown in Figure 7, the reflectivity of the evergreen needle forest is retrieved using Equation (5), which is conceived as an additional indicator to evaluate the performance of the proposed network. Figure 8 shows the comparison of the actual reflectivity spectra of the evergreen needle forest and our predicted results. The three atmospheric complete absorption bands approximately centered at $1.38 \mu \mathrm{m}, 1.88 \mu \mathrm{m}$, and $2.7 \mu \mathrm{m}$, respectively, are colored in white in Figure 8 . In these three atmospheric absorption bands, the target's reflectivity is not retrieved as expected. Other than that, the orange dots represents the mean of the 13 predicted reflectivity spectra using various elevation angles, while the green error bar delineates the standard deviation of the 13 predictions. It 

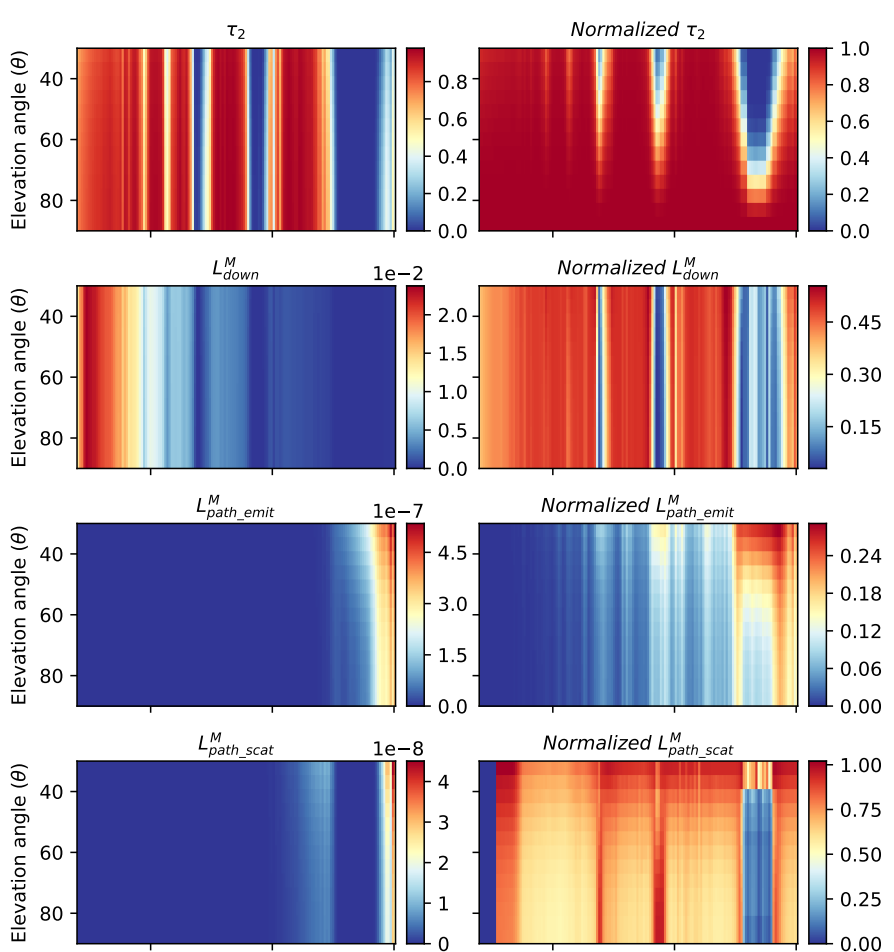

Normalized $L_{\text {path_scat }}^{M}$

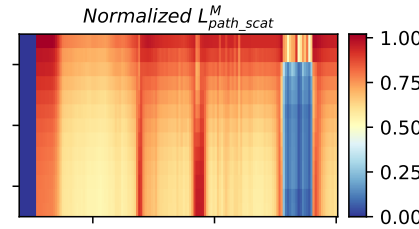

$L_{\text {solar_scat1 }}^{M}$

Normalized $L_{\text {solar_scat1 }}^{M}$
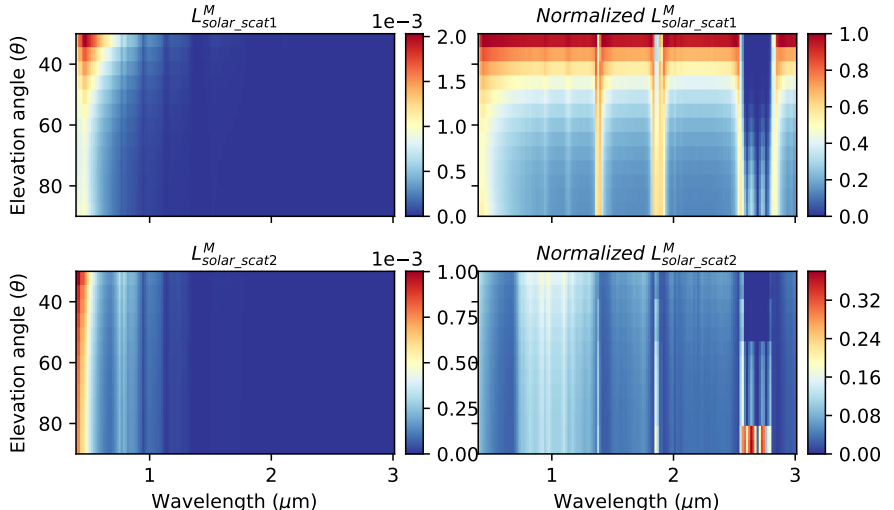

Figure 6: Matrix view of six solar and atmospheric radiative components for the evergreen needle forest and their normalizations. In the first column, from top to bottom, they are $\tau_{2}(\lambda), L_{d o w n}^{M}, L_{\text {path_emit }}^{M}, L_{\text {path_scat }}^{M}, L_{\text {solar_scat } 1}^{M}, L_{\text {solar_scat } 2}^{M}$, respectively, and their normalizations are aligned in the second column.

shows that the predicted reflectivity is well matched with the ground-truth (indicated in black dashed line). In addition, the small standard deviation demonstrates the high stability of the proposed network under multi-scan geometry setting.

\section{Discussion}

Our analysis shows that the proposed time-dependent network is capable of accurately providing atmospheric characteristics and target's spectral signature from multi-scan hyperspectral data with two temporal factors; its results 

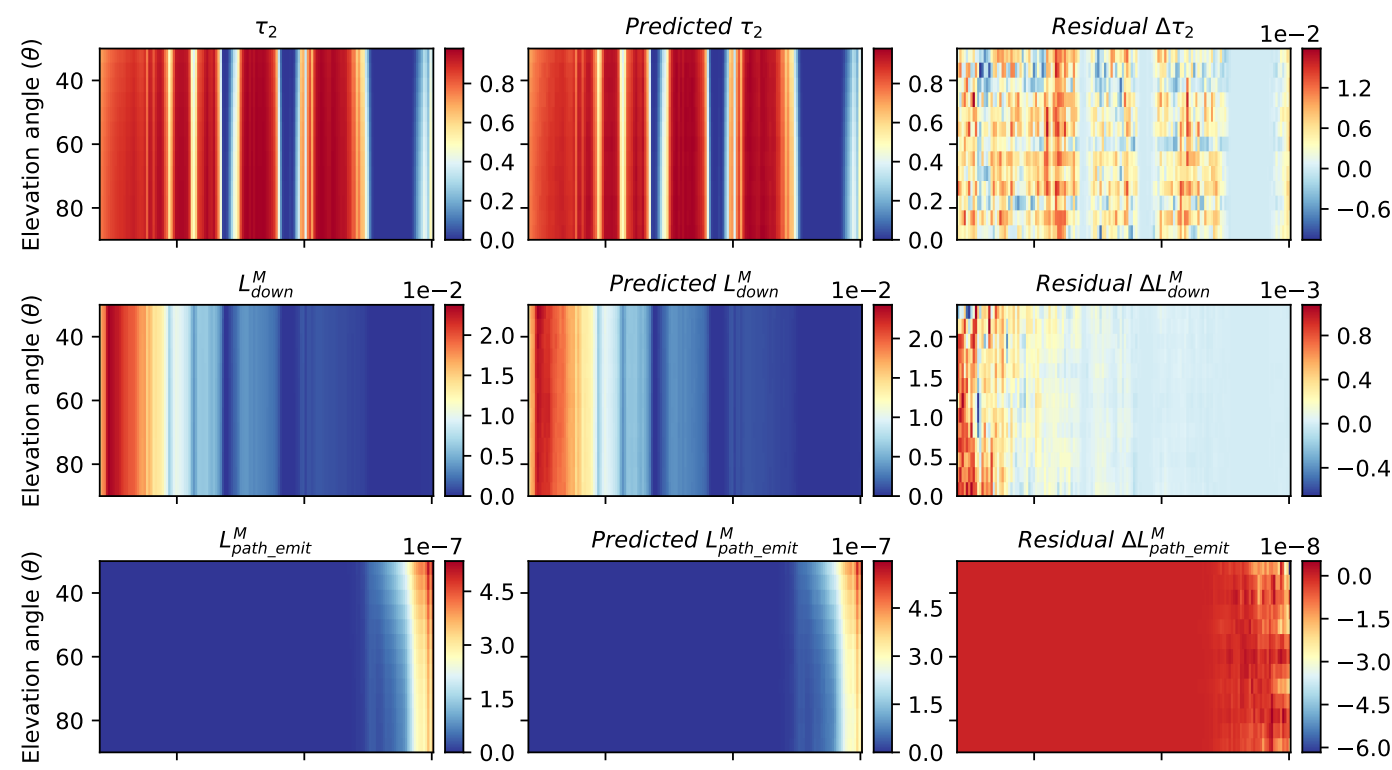

Residual $\Delta L_{\text {path emit }}^{M} \quad 1 e-8$
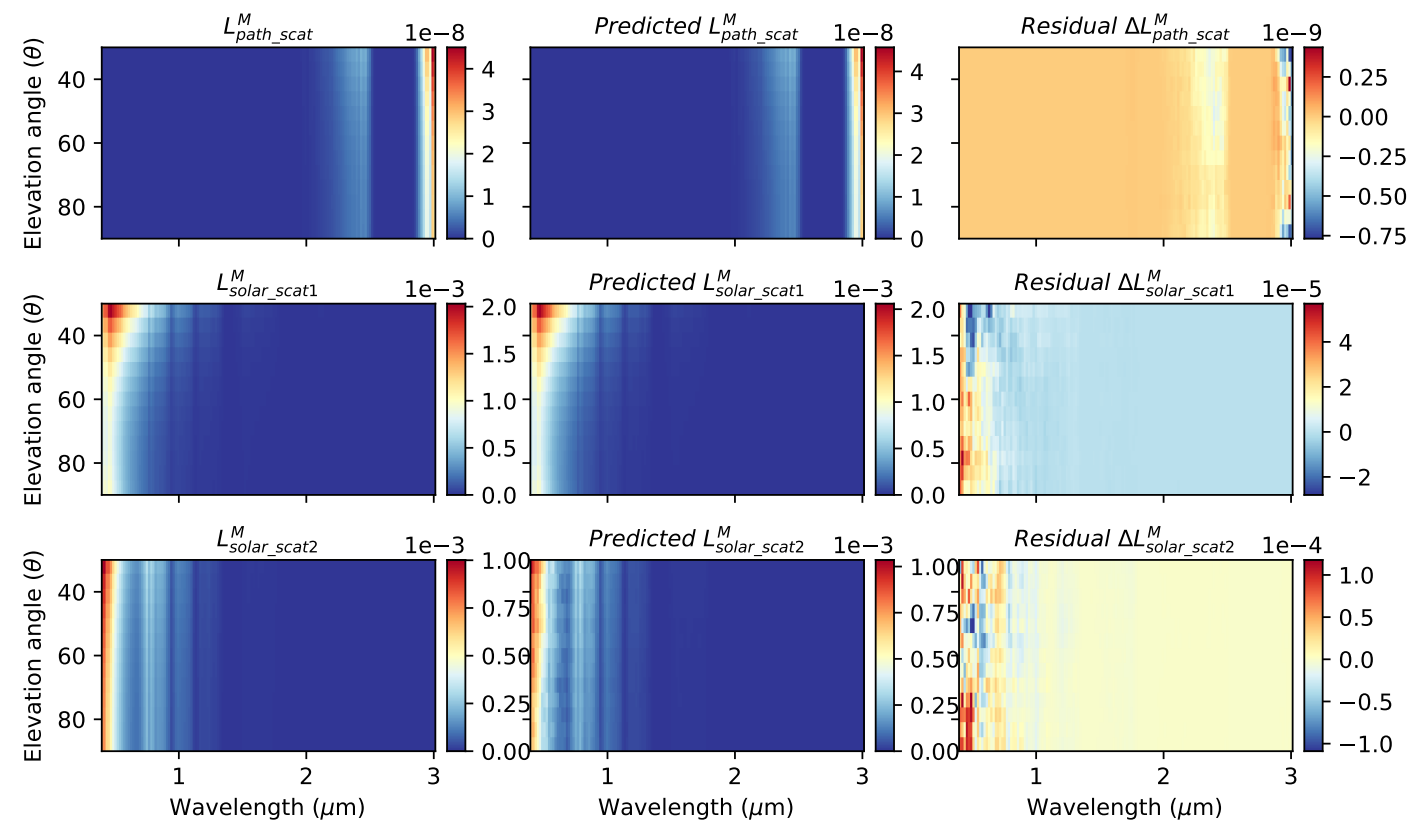

Figure 7: The comparison of six solar and atmospheric radiances of an evergreen needle forest collected at 2:00pm on January $10^{\text {th }}$ and our predicted results. From left to right in columns,they are the ground truth of six RTE components, our predicted results, and the residuals, respectively.

can be used to obtain a precise prediction of the target's reflectivity, which can be applied to real time atmospheric correction and target detection by comparing the predicted reflectivity with spectral signatures in database library.

However, more studies need to be made to understand the time dependency of the network. To study the contributions of two temporal factors in the final prediction, two experiments are carried out: 1) feeding the well trained network with a wrong day and a correct time factors for a specific target; 2) feeding the well trained network with a correct 


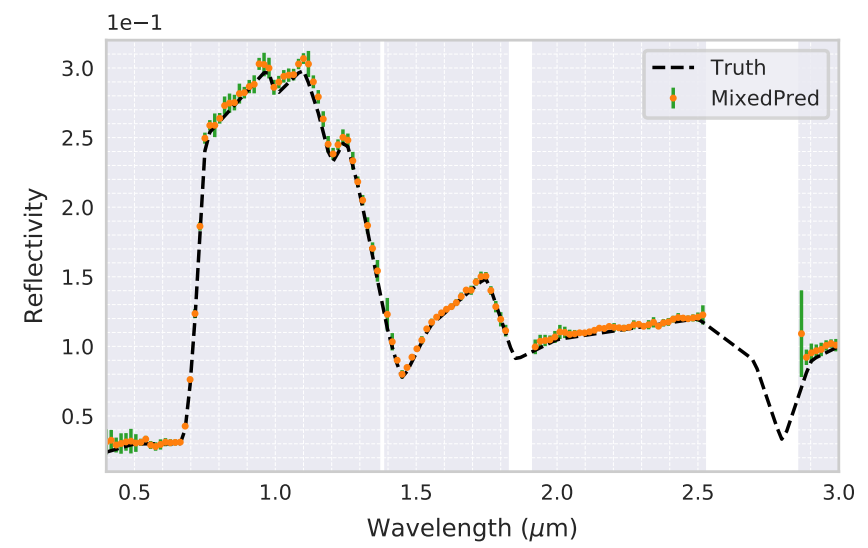

Figure 8: The comparison between the actual reflectivity (black dashed line) of the evergreen needle forest and our predicted results using the total radiance collected at $2: 00 \mathrm{pm}$ on January $10^{\text {th }}$. The orange dots represent the mean of 13 predicted reflectivities using different elevation angles, and the green error bar is the standard deviation of 13 predictions.

day and a wrong time factors for a specific target.

In the first experiment, we test the impact of the day factor on the network prediction. The hyperspectral at-sensor radiance map collected at 2:00pm on the $178^{\text {th }}$ day of the year (June $27^{\text {th }}$ ) is chosen as an example. The collected total radiance map and a correct time factor $(2: 00 \mathrm{pm})$ are repeatedly fed into the well trained network but with a day factor selected starting from 1 to 365 . After every evaluation, the mean absolute residuals between the predicted results and six normalized ground-truth components are calculated, and the result is plotted in Figure 9, where the orange line represents the mean absolute error (MAE) of residuals using 13 elevation angles with light blue colored area denoting the standard deviation of residuals with different elevation angles. In Figure 9, we observe that the mean error of the prediction reaches a minimum when the correct day factor, i.e., the $178^{\text {th }}$ day of the year, is fed into the network. However, as the input day factor moves away from the correct date, the predicted error increases, as well as its standard deviation. Furthermore, an approximately symmetric about the $178^{\text {th }}$ day is shown in Figure 9 , which can be explained by the approximate symmetrical atmospheric characteristics about summer. Therefore, it is reasonable to conclude that the proposed network has the ability of adapting to a diurnal changing atmospheric environment.

In the second experiment, we select eight total radiance maps for the evergreen needle forest collected at different times on the $178^{\text {th }}$ day of the year as input of the network, respectively. The correct day factor is imported with a randomly selected time value, from $6: 00 \mathrm{am}$ to $8: 00 \mathrm{pm}$ with a two hour interval. Similarly to the first experiment, after each evaluation, the mean absolute errors of the predicted results and six normalized ground-truth components are calculated along wavelength and elevation angle axes. The confusion matrix of MAE is displayed in Figure 10. As expected, the smallest predicted errors at each time are located on the main diagonal, and the largest errors are appeared at the farmost way of the correct time. The prediction error also increases as the coordinate moves away 


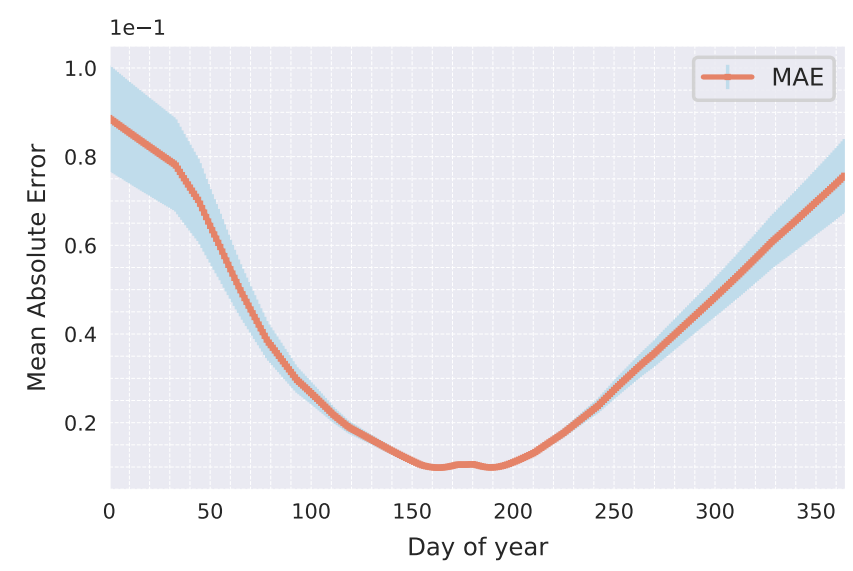

Figure 9: The error analysis of the day temporal factor by feeding a random day value into the network with the data collected at 2:00pm on the $178^{\text {th }}$ day of the year.

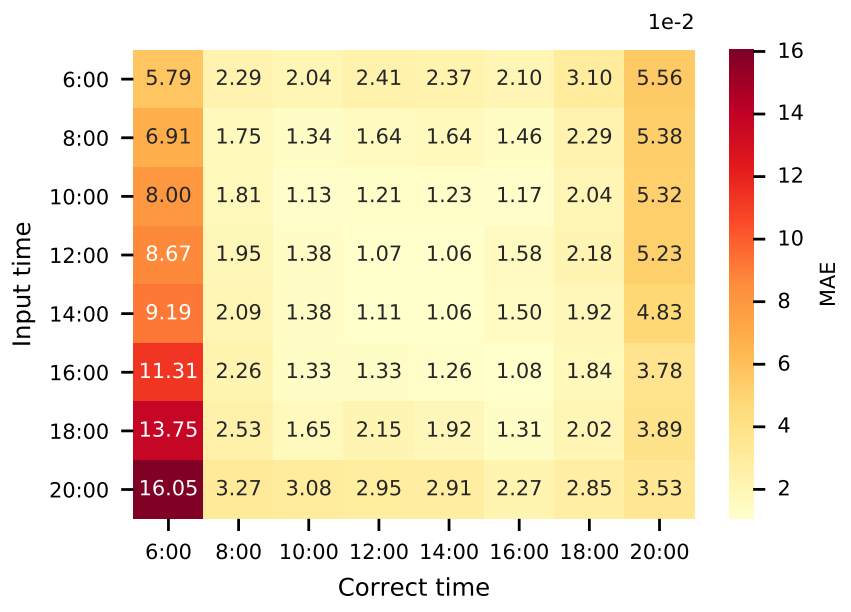

Figure 10: The error analysis of the time temporal factor by feeding a random time value into the network with data collected from 6:00 to 20:00 with two hour intervals on the $178^{\text {th }}$ day of the year.

from the main diagonal. These results suggest that our network is capable of accurately predicting atmospheric characteristics with an diurnally-and-hourly varying atmospheric environments. In addition, we observe that the MAE increases as the collection time moves away from noon along the main diagonal. This is possibly caused by the weak radiation at sunrise times which leads to the relative higher percentage errors comparing to data collected at noon. Though our experiment is conducted at the Pennsylvania State University, University Park, PA, USA, it is straightforward to be generalized to diurnally collected hyperspectral data at other locations.

To understand the importance of the 'mixed' training dataset, the proposed network is also trained separately with 15 vegetations and six gray bodies with varying constant reflectivities. These three networks are then applied to the test dataset including 27 materials separately. We extracted the predicted results for 24 materials at 2:00pm on January 

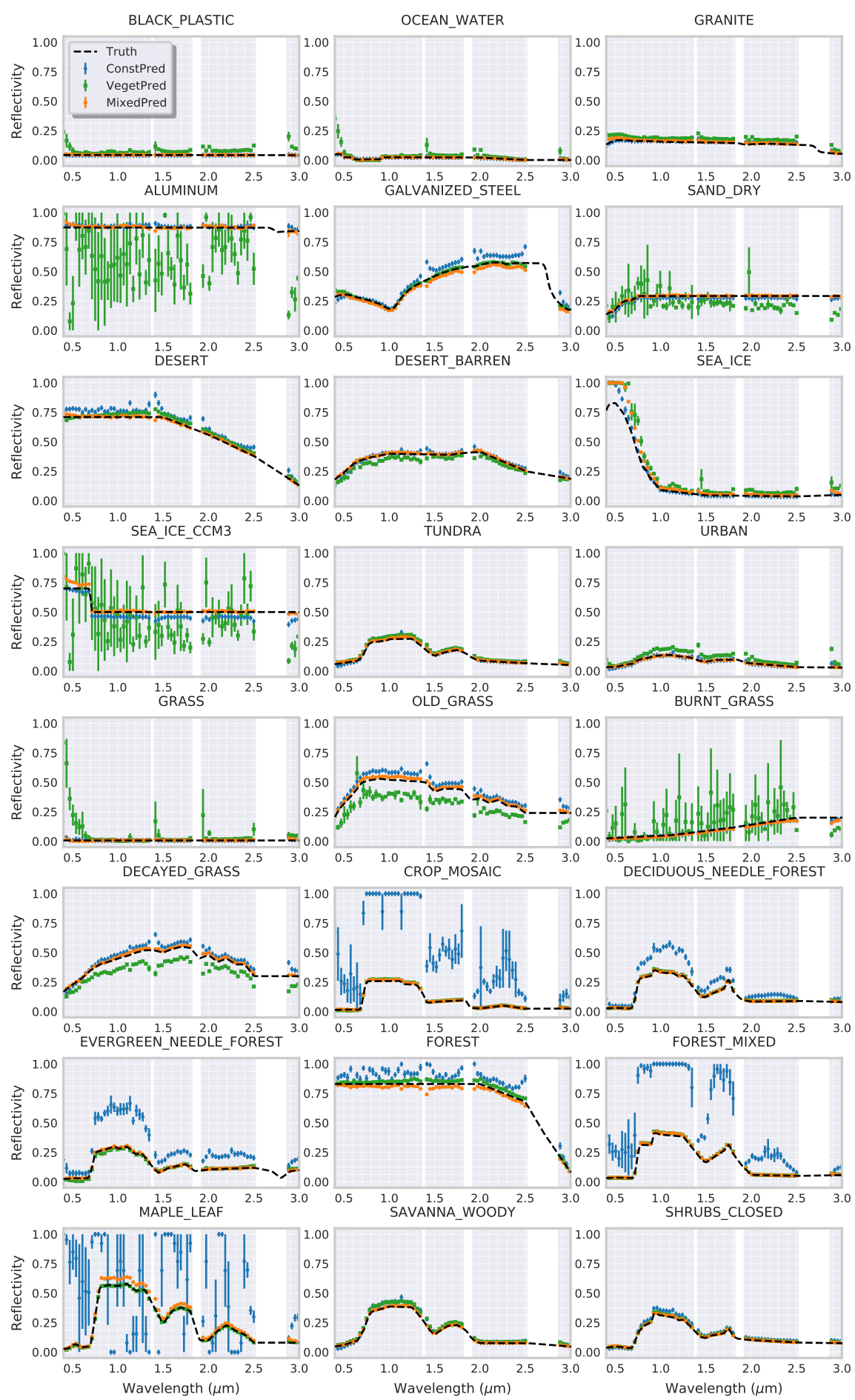

Figure 11: The comparison of the ground-truth reflectivities and predicted results using networks trained by three different datasets, with dashedblack line showing the ground-truth reflectivity spectra of the targets, blue showing predictions using network trained with six gray bodies, green showing predictions using network trained with 15 vegetations, orange showing predictions using network trained with a mixture of both. The dot (vertical line) represents the mean (standard deviation) of 13 predictions using different elevation angles. 
$10^{\text {th }}$ from the test dataset, and calculated the estimated reflectivities. The comparisons of the retrieved reflectivities and their ground-truths are shown in Figure 11. The dot represents the mean of 13 predicted reflectivity spectra with variant elevation angles and the vertical line is corresponding to the standard deviation of 13 predicted results. The dot/line color represents the estimated results from the network used, with blue showing the network trained with six gray bodies only; green showing the network trained with 15 vegetations only, and orange showing the network trained with a mixture of vegetation and six gray bodies.

The comparison shows that the network trained with six gray bodies is capable of retrieving the spectral signatures of the target that does not dramatically changes. However, it fails to predict most of vegetations' reflectivity spectra because of their complex spectral signatures. On the contrary, the network trained with 15 vegetations is able to precisely predict the spectral signatures of vegetations and most of other materials. However, its prediction for the materials with smoothly varying spectra, for instance, aluminum, sea ice, and burnt grass, has a very high standard deviation which implies a lower stability of the network's performance for these materials. In other words, for those with smooth spectral signatures, the prediction accuracy using the network trained with 15 vegetations is highly dependent to the elevation angle. The network trained with the 'mixed' training dataset outperforms both of these two separately trained networks on both vegetation and other materials with a negligible standard deviation. Thus, a mixture of vegetations and six gray bodies with different constant reflectivities is essential to improve and stabilize the performance of the network on a wide variety of targets.

Though the accuracy and capacity of our designed network is promising, one important practical consideration is that it only accepts a multi-scan hyperspectral data with no missing elevation angle $\left(30^{\circ}\right.$-to- $\left.90^{\circ}\right)$ or wavelength band $(0.4 \mu \mathrm{m}$-to- $3.0 \mu \mathrm{m})$. The standard deviation of the predicted error using different elevation angles is marginal, which is a positive indicator that the network may be stable for applications using data with missing elevation angles. The detailed investigation and compensation of applying the network with incomplete data is an ongoing research. Furthermore, settled geometry settings are assumed through our entire experiments and analysis, such as the unitary spatial resolution of the airborne sensor and the fixed observation range and azimuth. However, the application on the hyperspectral remote sensing data collected with different geometry settings can be implemented by finetuning our trained network based on a strategy of transfer learning.

\section{Conclusions}

The multi-scan hyperspectral remote sensing data collected by the new generations of agile sensors contains multiple spectral and temporal information within the full spatial dimensionality. This provides an opportunity for real time atmospheric correction and target detection by taking advantages of deep learning methods. In this paper, 
we designed a time-dependent neural network to estimate atmospheric characteristics and solar scattering radiances at a given time and day from the at-sensor total radiance. The results of our analysis show that the proposed network is able to accurately provide atmospheric characteristics at given times and precisely retrieve the reflectivity spectra of various materials, including vegetation and ocean. In addition, the retrieved reflectivity spectra using multi-scan hyperspectral data provide an approximation of the estimated error from perspectives of varying elevation angles. This approach is fully data-driven and well-suited for real time applications.

To investigate the time dependency of the proposed network, we introduce two experiments by feeding the network with incorrect temporal factors separately. The first experiment shows that the predicted error increases as the day factor moves away from the correct date. The prediction error has an approximate symmetric behavior which is consistent with the approximate symmetry of atmospheric characteristics about summer. With the first experiment, we may conclude that our network is able to precisely providing atmospheric characteristics and target detection under a daily changing environment. In the second experiment, our analysis shows that the predicted error significantly increases and its minimum located at the main diagonal corresponding to the correct time input. The second experiment confirms that the proposed network has the ability of accommodating a hourly varying atmospheric condition and providing precise predictions. In addition, the sensitivity tests have shown that in order to make accurate predictions for a wide variety of targets, it is critical to train the network with a mixture of targets including both vegetations and gray bodies with varying constant reflectivities. Though assumptions have been made on the geometry settings, real-time applications of such a time-dependent network on hyperspectral data from different collecting systems are possibly implemented by finetuning the network with a transfer learning strategy. In our future work, applying the network with incomplete data and real data will be discussed and analyzed.

\section{Acknowledgment}

This research was supported by the Defense Advanced Research Projects Agency (DARPA) award FA-865019-1-7905 and by the Penn State College of Earth and Mineral Sciences "Postdoc-Facilitated Innovation through Collaboration" award.

\section{References}

Adão, T., Hruška, J., Pádua, L., Bessa, J., Peres, E., Morais, R., Sousa, J.J., 2017. Hyperspectral imaging: A review on uav-based sensors, data processing and applications for agriculture and forestry. Remote Sensing 9, 1110

Adler-Golden, S.M., Matthew, M.W., Bernstein, L.S., Levine, R.Y., Berk, A., Richtsmeier, S.C., Acharya, P.K., Anderson, G.P., Felde, J.W., Gardner, J., et al., 1999. Atmospheric correction for shortwave spectral imagery based on modtran4, in: Imaging Spectrometry V, International Society for Optics and Photonics. pp. 61-69. 
Aspinall, R.J., Marcus, W.A., Boardman, J.W., 2002. Considerations in collecting, processing, and analysing high spatial resolution hyperspectral data for environmental investigations. Journal of Geographical Systems 4, 15-29.

Berk, A., Conforti, P., Kennett, R., Perkins, T., Hawes, F., Van Den Bosch, J., 2014. Modtran® 6: A major upgrade of the modtran® radiative transfer code, in: 2014 6th Workshop on Hyperspectral Image and Signal Processing: Evolution in Remote Sensing (WHISPERS), IEEE. pp. $1-4$.

Clark, R.N., King, T.V., 1987. Causes of spurious features in spectral reflectance data, in: Proceedings, Airborne Imaging Spectrometer Data Analysis Workshop, Jet Propulsion Laboratory, pp. 49-61.

Conel, J., Green, R., Vane, G., Bruegge, C., Alley, R., 1987. Airborne imaging spectrometer-2: Radiometry and a comparison of methods for the recovery of ground reflectance, in: Proceedings, Airborne Imaging Spectrometer Data Analysis Workshop, Jet Propulsion Laboratory, pp. $87-30$.

DiStasio Jr, R.J., Resmini, R.G., 2010. Atmospheric compensation of thermal infrared hyperspectral imagery with the emissive empirical line method and the in-scene atmospheric compensation algorithms: a comparison, in: Algorithms and Technologies for Multispectral, Hyperspectral, and Ultraspectral Imagery XVI, International Society for Optics and Photonics. p. 76952B.

Duan, M., Min, Q., Lü, D., 2010. A polarized radiative transfer model based on successive order of scattering. Advances in Atmospheric Sciences 27, 891-900.

Emde, C., Buras, R., Mayer, B., Blumthaler, M., 2010. The impact of aerosols on polarized sky radiance: model development, validation, and applications. Atmospheric Chemistry \& Physics 10.

Gao, B.C., Davis, C., Goetz, A., 2006. A review of atmospheric correction techniques for hyperspectral remote sensing of land surfaces and ocean color, in: 2006 IEEE International Symposium on Geoscience and Remote Sensing, IEEE. pp. 1979-1981.

Gao, B.C., Davis, C.O., 1997. Development of a line-by-line-based atmosphere removal algorithm for airborne and spaceborne imaging spectrometers, in: Imaging Spectrometry III, International Society for Optics and Photonics. pp. 132-141.

Gao, B.C., Heidebrecht, K.B., Goetz, A.F., 1993. Derivation of scaled surface reflectances from aviris data. Remote sensing of Environment 44, $165-178$.

Gao, B.C., Montes, M.J., Davis, C.O., Goetz, A.F., 2009. Atmospheric correction algorithms for hyperspectral remote sensing data of land and ocean. Remote Sensing of Environment 113, S17-S24.

Ghamisi, P., Yokoya, N., Li, J., Liao, W., Liu, S., Plaza, J., Rasti, B., Plaza, A., 2017. Advances in hyperspectral image and signal processing: A comprehensive overview of the state of the art. IEEE Geoscience and Remote Sensing Magazine 5, 37-78.

He, K., Zhang, X., Ren, S., Sun, J., 2016. Deep residual learning for image recognition, in: Proceedings of the IEEE conference on computer vision and pattern recognition, pp. 770-778.

Huang, G., Liu, Z., Van Der Maaten, L., Weinberger, K.Q., 2017. Densely connected convolutional networks, in: Proceedings of the IEEE conference on computer vision and pattern recognition, pp. 4700-4708.

Katkovsky, L.V., Martinov, A.O., Siliuk, V.A., Ivanov, D.A., Kokhanovsky, A.A., 2018. Fast atmospheric correction method for hyperspectral data. Remote Sensing 10, 1698.

Kingma, D.P., Ba, J., 2014. Adam: A method for stochastic optimization. arXiv preprint arXiv:1412.6980 .

Krizhevsky, A., Sutskever, I., Hinton, G.E., 2012. Imagenet classification with deep convolutional neural networks, in: Advances in neural information processing systems, pp. 1097-1105.

Kruse, F.A., 1988. Use of airborne imaging spectrometer data to map minerals associated with hydrothermally altered rocks in the northern grapevine mountains, nevada, and california. Remote Sensing of Environment 24, 31-51.

Manolakis, D., Pieper, M., Truslow, E., Lockwood, R., Weisner, A., Jacobson, J., Cooley, T., 2019. Longwave infrared hyperspectral imaging: 
Principles, progress, and challenges. IEEE Geoscience and Remote Sensing Magazine 7, 72-100.

Markelin, L., Simis, S.G., Hunter, P.D., Spyrakos, E., Tyler, A.N., Clewley, D., Groom, S., 2017. Atmospheric correction performance of hyperspectral airborne imagery over a small eutrophic lake under changing cloud cover. Remote Sensing 9, 2.

Mayer, B., 2009. Radiative transfer in the cloudy atmosphere, in: EPJ Web of Conferences, EDP Sciences. pp. 75-99.

Qu, Z., Kindel, B.C., Goetz, A.F., 2003. The high accuracy atmospheric correction for hyperspectral data (hatch) model. IEEE Transactions on Geoscience and Remote Sensing 41, 1223-1231.

Roberts, D., Yamaguchi, Y., Lyon, R., 1986. Comparison of various techniques for calibration of ais data. NASA STI/Recon Technical Report N $87,21-30$.

Ronneberger, O., Fischer, P., Brox, T., 2015. U-net: Convolutional networks for biomedical image segmentation, in: International Conference on Medical image computing and computer-assisted intervention, Springer. pp. 234-241.

Schott, J.R., 2007. Remote sensing: the image chain approach. Oxford University Press on Demand.

Siewert, C., 2000. A discrete-ordinates solution for radiative-transfer models that include polarization effects. Journal of Quantitative Spectroscopy and Radiative Transfer 64, 227-254.

Simonyan, K., Zisserman, A., 2014. Very deep convolutional networks for large-scale image recognition. arXiv preprint arXiv:1409.1556 .

Szegedy, C., Liu, W., Jia, Y., Sermanet, P., Reed, S., Anguelov, D., Erhan, D., Vanhoucke, V., Rabinovich, A., 2015. Going deeper with convolutions, in: Proceedings of the IEEE conference on computer vision and pattern recognition, pp. 1-9.

Szegedy, C., Vanhoucke, V., Ioffe, S., Shlens, J., Wojna, Z., 2016. Rethinking the inception architecture for computer vision, in: Proceedings of the IEEE conference on computer vision and pattern recognition, pp. 2818-2826.

Teke, M., Deveci, H.S., Haliloğlu, O., Gürbüz, S.Z., Sakarya, U., 2013. A short survey of hyperspectral remote sensing applications in agriculture, in: 2013 6th International Conference on Recent Advances in Space Technologies (RAST), IEEE. pp. 171-176.

Xu, F.X., Cervonea, G., Franchb, G., Salvador, M., 2020. Multiple geometry atmospheric correction for image spectroscopy using deep learning. Under Review x, $\mathrm{x}$.

Yu, S., Jia, S., Xu, C., 2017. Convolutional neural networks for hyperspectral image classification. Neurocomputing $219,88-98$. 